\title{
Hydrogen Permeation Characteristics of TiN-Coated
}

\section{Stainless Steels}

\author{
Motonori Tamura \\ Center for Industrial and Governmental Relations, The University of Electro-Communications, Tokyo 182-8585, Japan
}

\begin{abstract}
TiN films consisting of fine crystal grains, with diameters of about $100 \mathrm{~nm}$ or less, provided superior hydrogen-permeation barriers. Applying a coating of TiN on a stainless steel substrate reduced the hydrogen permeation by a factor of about 100 to 5,000 compared with uncoated substrates. Effect of microstructure of films on hydrogen-permeation behavior is studied. The test specimens coated with films, with columnar crystals grown vertically on the substrate, tended to exhibit higher hydrogen permeability. The grain boundaries of the films became trap sites for hydrogen and microcrystalline structures with many grain boundaries are expected to provide effective hydrogen-barrier performance.
\end{abstract}

Key words: Hydrogen permeation, TiN coating, microstructure, crystal grain, stainless steel.

\section{Introduction}

There have been attempts in recent years to utilize hydrogen gas as an alternative to fossil fuel, leading to the development of numerous hydrogen-energy systems that use high-pressure hydrogen. Molecular hydrogen dissociates relatively easily to form hydrogen atoms on the surface of steel when exposed to a hydrogen gas atmosphere, and hydrogen permeation in steel has been reported to cause hydrogen embrittlement [1-8].

Studies concerning hydrogen-barrier films have been conducted in a variety of fields such as nuclear fusion reactors, fuel cells, $\mathrm{H}_{2} \mathrm{~S}$ corrosion components, or vacuum equipment. Dense ceramic films such as $\mathrm{Al}_{2} \mathrm{O}_{3}, \mathrm{TiC}, \mathrm{TiN}$, and $\mathrm{BN}$ have been reported to provide hydrogen-barrier performance. The hydrogen-permeation reduction factors vary significantly from one report to another, however. The variance for $\mathrm{Al}_{2} \mathrm{O}_{3}$ is between 10 and 10,000 [9], for example, owing to significant variations in hydrogen permeation behavior with the films' microstructures. Many researchers have experimented with the forming

\footnotetext{
Corresponding author: Motonori Tamura, Ph.D., professor, research field: metallurgical coatings. E-mail: mtamura@sangaku.uec.ac.jp.
}

conditions to vary the microstructure of films. For instance, in Thornton's structure zone model, microstructures are classified according to factors such as the substrate temperature and argon pressure during film formation [10].

This study focused on the impact of differences in thin-film microstructures on hydrogen permeation behavior.

\section{Experimental Setup}

\subsection{Deposition Process and Characterization of Films}

Type "316L" austenitic stainless steel was used for the substrates and TiN films were formed on one side of such substrates by RF sputtering. RF sputtering was performed by the power of 600 to $3,000 \mathrm{~W}$ in order to obtain variety of microstructures of TiN films. A film thickness of 1.8 to $2.0 \mu \mathrm{m}$ was obtained. The film thickness, film cross-sections, and microstructures were examined with a SEM (Scanning electron microscope); the crystalline phase of was analyzed by XRD (X-ray diffraction); the chemical composition of the films were analyzed by EPMA (Electron probe micro-analyzer); the sizes of the crystal grains were analyzed with a SPM (Scanning probe microscope) and XTEM (X-ray transmission electron microscope). 


\subsection{Hydrogen-Permeation Tests}

Hydrogen-permeation tests were performed on the coated stainless steel samples. These tests were based on the differential-pressure methods [11]. Fig. 1 shows a schematic illustration of the apparatus. This part of the standard specifies the use of a pressure sensor or a gas chromatograph for determining the gas-transmission rate of a single-layered plastic film or sheet and multi-layered structures under a differential pressure. A gas chromatograph was used in this study. The samples (diameter: $35 \mathrm{~mm}$; thickness: $0.1 \mathrm{~mm}$ ) were set on a susceptor, which could be held in place at temperatures up to $773 \mathrm{~K}$. The apparatus was evacuated to $10^{-6} \mathrm{~Pa}$. After the test temperature stabilized, hydrogen (purity of 99.995\%) was introduced into the susceptor side of the chamber at a filling pressure of $400 \mathrm{kPa}$. The stainless-steel-sample side of the apparatus was continuously evacuated. The permeation area was $6.6 \mathrm{~cm}^{2}$.

The permeability $\phi$, is defined by the expression:

$$
\phi=(\mathrm{J} d) /\left(\mathrm{A}(\Delta \mathrm{p})^{\mathrm{n}}\right),
$$

where $\mathrm{J}$ is the permeation flux of hydrogen through a sample of area A and thickness $d$, under a partial pressure gradient $\Delta \mathrm{p}$ across the sample called the driving pressure. The exponent $n$ represents different permeation regimes: diffusion-limited and surface-limited when $n=0.5$ and 1 respectively.
Hydrogen permeation through a thin-film-coated steel sample is known to be diffusion-limited when the driving pressure is between $10^{4}$ and $10^{5} \mathrm{kPa}$ [12].

The permeation flux was continuously measured. After $30 \mathrm{~min}$, for example, the standard deviation decreased below $10 \%$ among the data for permeation flux through the non-coated stainless steel substrate at $773 \mathrm{~K}$ under a hydrogen pressure of $400 \mathrm{kPa}$.

\section{Results and Discussion}

\subsection{Hydrogen-Permeation Mode}

The permeation of hydrogen through solid materials proceeds via adsorption, dissociation, diffusion, and recombination coupled with desorption. Fig. 2 shows a schematic illustration of hydrogen permeation in coated samples.

A hydrogen molecule $\left(\mathrm{H}_{2,}, \mathrm{ad}\right)$ is adsorbed on the surface of the film and decomposes to hydrogen atoms $\left(\mathrm{H}_{\mathrm{ad}}\right)$. Such adsorbed hydrogen atoms diffuse into the film from the film surface $\left(\mathrm{H}_{\text {film, in }}\right)$ and move toward the interface with the substrate $\left(\mathrm{H}_{\text {film, out }}\right)$. Hydrogen atoms diffuse into the substrate from the interface of the film $\left(\mathrm{H}_{\text {sub, in }}\right)$ to the non-coated side of the substrate $\left(\mathrm{H}_{\text {sub, out }}\right)$. At the non-coated side of the substrate, the hydrogen atoms $\left(\mathrm{H}_{\mathrm{des}}\right)$ form hydrogen molecules and desorption occurs. In this study, a driving force of hydrogen permeation was the pressure difference

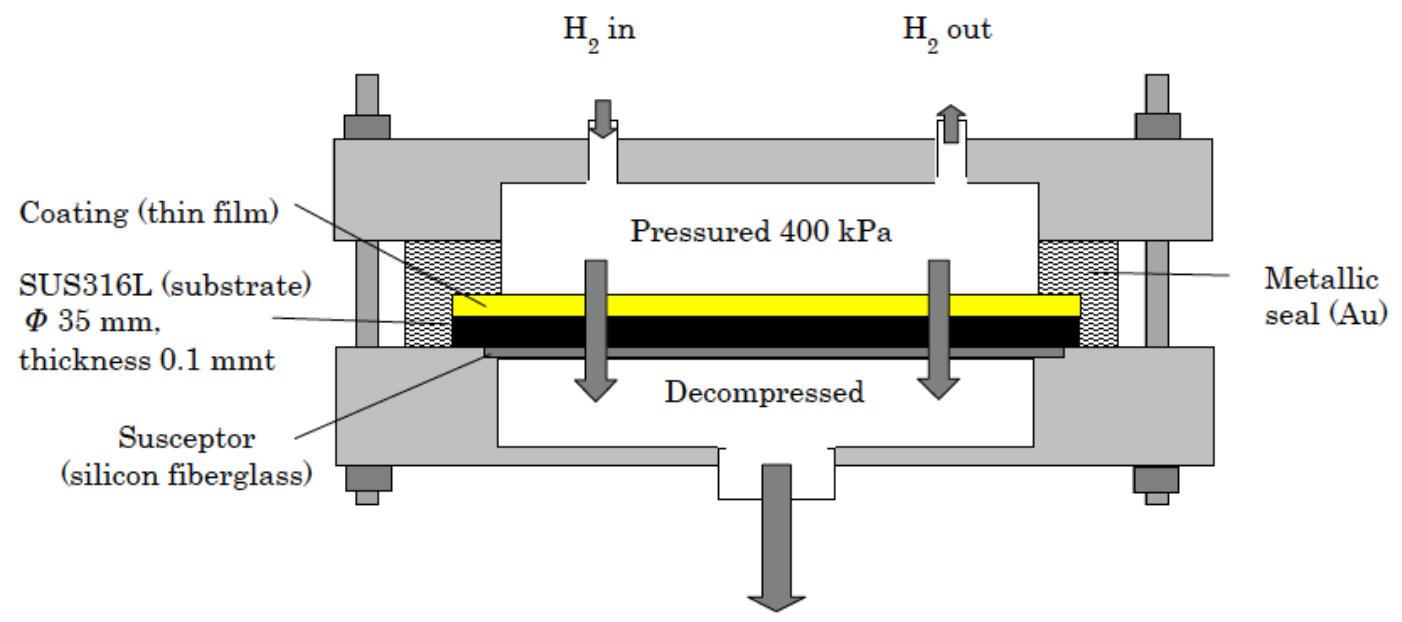

Measured by gas chromatography

Fig. 1 Apparatus for hydrogen-permeation tests. 


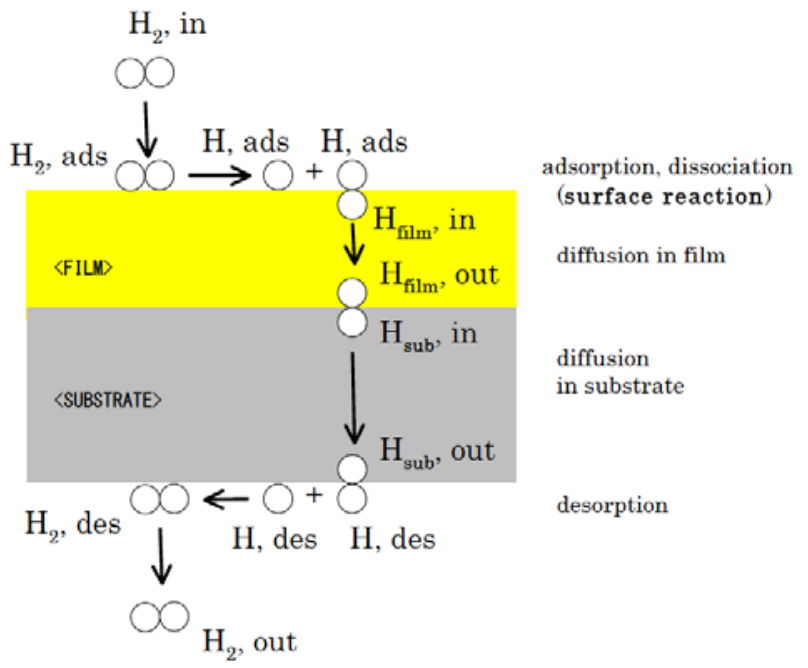

Fig. 2 Hydrogen-permeation mechanism.

between the high and low pressure sides. Hydrogen permeation occurred in the film and the substrate because of decompression in the gas phase at the non-coated side of the substrate.

Our previous study [13] has demonstrated the dependence of permeation flux $\mathrm{J}$ on the driving pressure $\Delta \mathrm{p}$ in $\mathrm{BN}-$ and TiN-coated samples. The exponent $n$ had a value of $0.48 \sim 0.53$ at $573 \sim 773 \mathrm{~K}$, which indicated that hydrogen passed through the samples in the diffusion-limited permeation mode. When $n=0.5$ (diffusion-limited regime) in Eq. (1), the overall permeation flux of hydrogen through the sample can be given by Fick' $\mathrm{s}$ law. In a steady state, the overall permeation flux $\mathrm{J}$ (measured in $\mathrm{mol} \cdot \mathrm{s}^{-1}$ ) through a sample of thickness $d_{\text {film }}+d_{\text {sub }}\left(d_{\text {film }}\right.$, thickness of film: $1.5 \times 10^{-6} \mathrm{~m}$; $\mathrm{d}_{\text {sub }}$, thickness of substrate: $\left.1.0 \times 10^{-4} \mathrm{~m}\right)$ and area $\mathrm{A}\left(6.6 \times 10^{-4} \mathrm{~m}^{2}\right)$ is expressed as:

$$
\mathrm{J}=\phi \mathrm{A}\left(\left(\mathrm{P}_{\mathrm{H} 2, \text { in }}\right)^{0.5}-\left(\mathrm{P}_{\mathrm{H} 2, \text { out }}\right)^{0.5}\right) /\left(\mathrm{d}_{\text {film }}+\mathrm{d}_{\mathrm{sub}}\right)
$$

where, $\phi$ is the permeability of the sample as shown before, and $\mathrm{P}_{\mathrm{H} 2 \text {, in }}\left(4.0 \times 10^{5} \mathrm{~Pa}\right)$ and $\mathrm{P}_{\mathrm{H} 2 \text {, out }}\left(1.0 \times 10^{-6} \mathrm{~Pa}\right)$ are the hydrogen pressures at the feed side and at the vacuum-pumping side respectively.

\subsection{Effect of Microstructure of Coatings on Hydrogen-Permeation Behavior}

Fig. 3 shows the XRD pattern of TiN film. EPMA data showed that chemical compositions of $\mathrm{Ti}$ in films were 47 to 51 atomic percent.

The test specimens with TiN coatings underwent a significant change in hydrogen permeability as shown in Fig. 4, which depended on the test temperature, and the decline in the hydrogen permeability was greater at lower temperatures. Since the hydrogen permeability is a reflection of the diffusion of hydrogen in the film, the diffusion coefficient of hydrogen should also decrease with lower temperature in TiN.

The relationship between the crystalline grain size and hydrogen permeability at $773 \mathrm{~K}$ is shown in Fig. 5. Test specimens covered with films consisting of smaller crystalline grains tended to have lower hydrogen permeability.

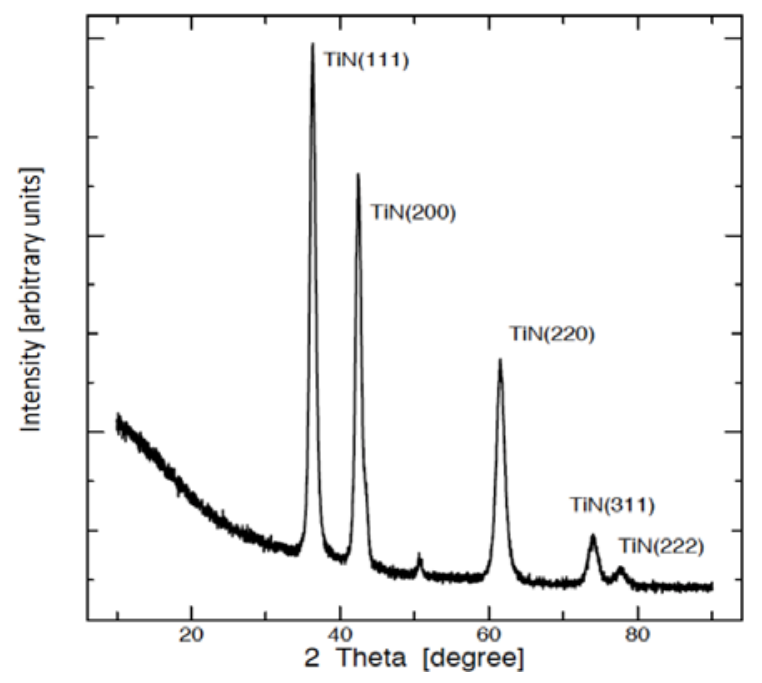

Fig. 3 XRD pattern of TiN film.

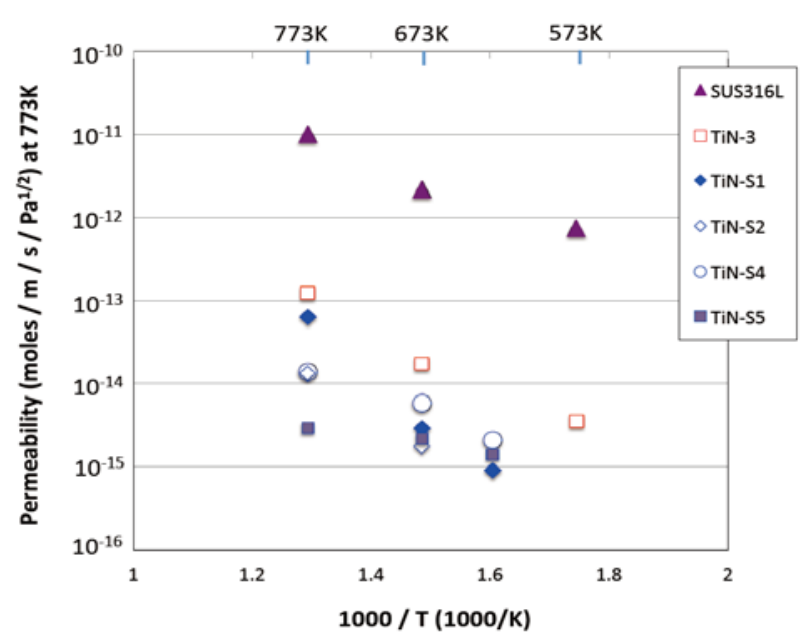

Fig. 4 Hydrogen-permeation performance. 


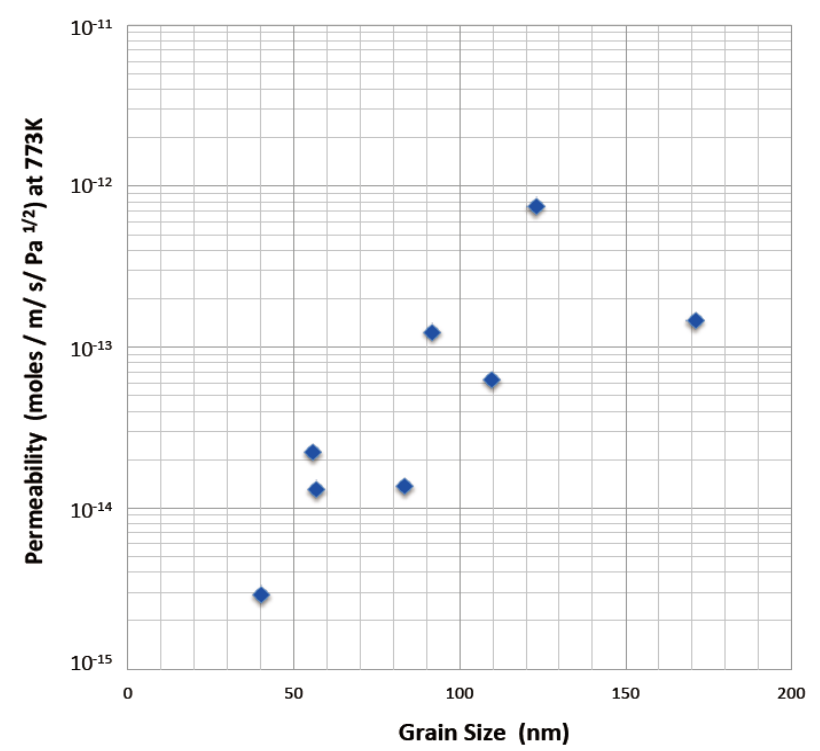

Fig. 5 Effect of grain size on hydrogen-permeation.

TiN coatings (TiN-S1, TiN-S2, TiN-S4, TiN-S5), which were deposited by sputtering, composed of microcrystalline grains equivalent to those in Zone $\mathrm{T}$ of Thornton's structure zone model [10], while the columnar TiN-3 grains, which were deposited by ion plating, were equivalent to those in Zone II (columnar). There were differences in the crystalline grain sizes, with higher hydrogen permeability for the columnar TiN-3 grains with relatively larger size (> $100 \mathrm{~nm}$ ). The TiN films with columnar structures tended to have relatively high hydrogen permeability. Films consisting of microcrystalline grains equivalent to those in Zone T of Thornton's structure zone model, were confirmed to have higher hydrogen-barrier performance. Many grain boundaries existed within the films, which could potentially present some form of obstruction when hydrogen passed through.

Studies concerning the state of hydrogen in materials cited lattice defects (atomic vacancy, dislocation, and grain boundaries), impurity atoms, precipitates, inclusion boundaries, voids, etc., as hydrogen trap sites. When the microstructure of examined materials was fine-grained, high density of dislocations and numerous grain boundaries provided longer travel of atomic hydrogen through a crystal lattice of examined materials and slower breakdown of hydrogen at the opposite side of membrane [12]. Hydrogen solid solubility of a fine-grained crystalline $\mathrm{Ni}$ test specimen was known to vary significantly from that of a polycrystalline test specimen of Ni. The hydrogen content in Ni depended on the crystalline grain size [14]. It had been pointed out that the hydrogen diffusion coefficient in some steel depends on the crystalline grain size and the diffusion coefficient decreases when the crystalline grain size was small [15]. These findings led us to believe that the effects of grain boundaries could not be ignored when the diffusion rate of hydrogen was relatively slow.

Hydrogen diffusion in steel has been known to be a few orders of magnitude lower in materials with ion covalent bonds, such as ceramic films [16-18], and the hydrogen trap sites are believed to function sufficiently. The micro-scale of a film's crystal grains is believed to have caused the grain boundaries to act as effective hydrogen-diffusion barriers.

\section{Conclusions and Future Directions}

TiN films consisting of fine crystal grains, with diameters of about $100 \mathrm{~nm}$ or less, provided superior hydrogen-permeation barriers. Applying a coating of TiN on a stainless steel substrate reduced the hydrogen permeation by a factor of about 100 to 5,000 compared with uncoated substrates. The grain boundaries of the films became trap sites for hydrogen and microcrystalline structures with many grain boundaries are expected to provide effective hydrogen-barrier performance.

Extensive studies of the correlation between film structure and deposition parameters have been carried out over the past ten decades. From an understanding of film formation, follows the possibility for micro-structural and nano-structural engineering in order to design a material for specific technological applications. This has led to the development and refinement of Thornton's structure zone models that systematically categorize self-organized structural evolution during physical vapor deposition as a 
function of film growth parameters. The first structure zone models were derived from relatively low-resolution of optical and SEM observations. Later, cross-sectional TEM and SPM analyses were employed to provide more detailed structural characterization.

In this study, behavior of hydrogen permeability of coated stainless steels was analyzed with a focus on the grain size of TiN films. Thin films usually exhibit a wide variety of microstructures characterized in terms of not only grain size and but also crystallographic orientation, lattice defects, phase composition, and surface morphology. Further study is necessary with a focus on the various microstructures to understand the correlation between the hydrogen permeation behavior and film microstructure, and to develop high-performance hydrogen barrier coatings.

\section{References}

[1] Beachem, C. D. 1972. "A New Model for Hydrogen-Assisted Cracking (Hydrogen 'Embrittlement').” Metall. Trans. 3: 437-51.

[2] Hirth, J. P. 1980. "Effects of Hydrogen on the Properties of Iron and Steel", Metall. Trans. A 11: 861-90.

[3] Nagumo, M., Nakamura, M. and Takai, K. 2001. "Hydrogen Thermal Desorption Relevant to Delayed-Fracture Susceptibility of High-Strength Steels." Metall. Trans. A 32: 339-347.

[4] Takai, K., Homma, Y., Izutsu, K. and Nagumo, M. 1996. "Identification of Trapping Sites in High-Strength Steels by Secondary Ion Mass Spectrometry for Thermal Desorbed Hydrogen.” J. Jpn. Inst. Met. 60 (12): 1155-62.

[5] Fukuyama, S., Yokogawa, K., Kubo, K. and Araki, M. 1985. "Fatigue Properties of Type 304 Stainless Steel in High Pressure Hydrogen at Room Temperature." Trans. Jpn. Int. Met. 26: 325-31.

[6] Han, G., He, J., Fukuyama, S. and Yokogawa, K. 1998. "Effect of Strain-Induced Martensite on Hydrogen Environment Embrittlement of sSensitized Austenitic Stainless Steels at Low Temperature." Acta Mater 46: 4559-70.
[7] Marchi, C. S., Somerday, B. P. and Robinson, S. L. 2007. "Permeability, Solubility and Diffusivity of Hydrogen Isotopes in Stainless Steels at High Gas Pressures." Int. J. Hydrogen Energ. 32: 100-16.

[8] Tamura, M. and Shibata, K. 2005. "Evaluation of Mechanical Properties of Metals at $45 \mathrm{MPa}$ Hydrogen." J. Jpn. Inst. Met. 69: 1039-48.

[9] Hollenberg, G. W., Simonen, E. P., Kalinin, G. and Terlain, A. 1995. "Tritium/Hydrogen Barrier Development.” Fusion Eng. Des. 28: 190-208.

[10] Thornton, J. A. 1974. "Influence of Apparatus Geometry and Deposition Conditions on the Structure and Topography of Tshick Sputtered Coatings.” J. Vac. Sci. Technol. 11: 666-70.

[11] Stem, S. A. 1968. J. Polym. Sci. 6: A-2.

[12] Chikada, T., Suzuki, A., Yao, Z., Levchuk, D., Mainer, H. and Terai, T. et al. 2009. "Surface Behavior in Deuterium Permeation through Erbium Oxide Coating." Fusion Eng. Des. 84: 590-2.

[13] Tamura, M., Noma, M. and Yamashita, M. 2014. "Characteristic Change of Hydrogen Permeation in Stainless Steel Plate by BN Coating." Surf. Coat. Tech. 260: 148-54.

[14] Hadzipašic, A. B., Malina, J. and Malina, M. 2011. "The Influence of Microstructure on Hydrogen Diffusion and Embrittlement of Multiphase Fine-grained Seels with Increased Plasticity and Strength." Chem. Biochem. Eng. Q. 25 (2): 159-69.

[15] Oudriss, A., Creus, J., Bouhattate, J., Conforto, E., Berziou, C. and Savall, C. 2012. "Grain Size and Grain-Boundary Effects on Diffusion and Trapping of Hydrogen in Pure Nickel." Acta. Mater. 60: 6814-28.

[16] Yamabe, J., Matsuoka, S. and Murakami, Y. 2013. "Surface Coating with a High Resistance to Hydrogen Entry under High-Pressure Hydrogen-Gas Environment." Int. J. Hydrogen Energ. 38: 10141-54.

[17] Checchetto, R., Bonelli, M., Gratton, L. M., Miotello, A., Sabbioni, A. and Guzman, L. et al. 1996. "Analysis of the Hydrogen Permeation Properties of TiN-TiC Bilayers Deposited on Martensitic Stainless steel." Surf. Coat. Tech. 83:40-4.

[18] Sorieul, S., Miro, S., Taillades-Jacquin, M., Dailly, J., Mauvy, F. and Berger, M.-H. 2008. "Hydrogen Diffusion in High Temperature Proton Conducting Ceramics." Nuclear Instruments and Methods in Physics Research Section B: Beam Interaction and Atoms 266, 8: 1430-3. 